\title{
Friedens- und Konfliktforschung in der Schweiz: ein facettenreiches Patchwork
}

\section{Laurent Goetschel \& Sara Hellmüller}

\section{Zeitschrift für Friedens- und Konfliktforschung}

ZeFKo Studies in Peace and Conflict

ISSN 2192-1741

Volume 8

Number 1

Z Friedens und Konflforsch (2019) 8:79-98

DOI 10.1007/s42597-019-00011-2
ZeFKo

Zeitschrift für Friedensund Konfliktforschung

ZeFKo Studies in Peace and Conflict

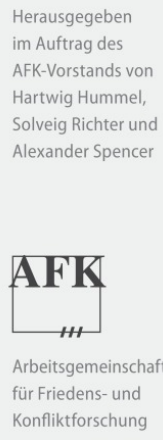

uffätze

Ulrika Mientus

Normalität und verweigerte Opferschaft in Transitional Justice Prozessen: Die späte und begrenzte Anerkennung von „Euthanasie“ und Zwangssterilisation als NS-Unrecht

Hendrik Hegemann

Die Politik der Radikalisierung: Ein politisches Narrativ zwischen Komplexitätsreduzierung und Selbstvergewisserung

Literaturbericht

Friedens- und Konfliktforschung in der Schweiz: ein facettenreiches Patchwork

Forumsbeiträge

Daniel Lambach. Patricia Schneider

Frieden studieren - und was dann? Ergebnisse einer Absolvent ${ }^{*}$ innenbefragung von Masterstudiengängen de Friedens- und Konfliktforschung

Andreas Nölke

Frieden und Zwang: Eine Kritik des neue Forschungsprogramms der HSFK aus der Sicht der Internationalen Politischen Ökonomie 
Your article is protected by copyright and all rights are held exclusively by Arbeitsgemeinschaft für Friedens- und Konfliktforschung e.V. (AFK) und die Autoren. This e-offprint is for personal use only and shall not be self-archived in electronic repositories. If you wish to self-archive your article, please use the accepted manuscript version for posting on your own website. You may further deposit the accepted manuscript version in any repository, provided it is only made publicly available 12 months after official publication or later and provided acknowledgement is given to the original source of publication and a link is inserted to the published article on Springer's website. The link must be accompanied by the following text: "The final publication is available at link.springer.com". 


\title{
Friedens- und Konfliktforschung in der Schweiz: ein facettenreiches Patchwork
}

\author{
Laurent Goetschel $(\mathbb{D} \cdot$ Sara Hellmüller $\mathbb{D}$
}

Online publiziert: 17. Juli 2019

(C) Arbeitsgemeinschaft für Friedens- und Konfliktforschung e.V. (AFK) und die Autoren 2019

Zusammenfassung Der Beitrag vermittelt einen Überblick zur Friedensforschung in der Schweiz. Er analysiert die erbrachten Forschungsleistungen historisch und institutionell und zeigt ihre facettenreichen disziplinären, methodologischen und thematischen Ausrichtungen auf. Dabei verdeutlicht er sowohl ihre internationale Anschlussfähigkeit wie auch ihr besonderes Profil. Letzteres beruht auf ihrem Verhältnis zur Schweizer Aussen- und Sicherheitspolitik und insbesondere zum Konzept der Neutralität. Die gezielte Förderung von Nord-Süd-Partnerschaften sowie die Praxisnähe gehören zu den wichtigen Merkmalen der Schweizer Friedensforschung. Der Artikel geht auch auf die epistemologische Verortung der Friedensforschenden in der Schweiz ein und schliesst mit Anregungen für zukünftige Forschungsvorhaben.

Schlüsselwörter Friedensforschung · Konfliktforschung · Friedensförderung · Neutralität · Schweiz

\section{Peace and Conflict Studies in Switzerland: a colorful patchwork}

Abstract This article provides an overview of Swiss peace research. It analyzes peace research undertaken in Switzerland from a historical and institutional perspective and demonstrates its multifaceted disciplinary, methodological and thematic approaches. Thereby, it highlights its international embedment as well as its specific profile. The uniqueness of Swiss peace research stems from its link to Swiss foreign and security policy and particularly the concept of neutrality. Important characteristics of Swiss peace research are the promotion of North-South partnerships and

L. Goetschel $(\bowtie) \cdot$ S. Hellmüller

swisspeace, Universität Basel, Basel, Schweiz

E-Mail: laurent.goetschel@swisspeace.ch

S. Hellmüller

E-Mail: sara.hellmueller@swisspeace.ch 
its practice relevance. The article also provides insights into the epistemological background of peace researchers in Switzerland and concludes with suggestions for future research.

Keywords Peace research · Conflict research · Peacebuilding · Neutrality · Switzerland

\section{Einleitung}

Wir wollen mit diesem Beitrag einen Überblick zur Friedensforschung in der Schweiz vermitteln. Diese sehen wir als Forschung, die sich mit den Ursachen von bewaffneten Konflikten und deren Bewältigung beschäftigt (Brühl 2012; Brzoska 2012; Müller 2012; Hagmann 2014; Cooper und Finley 2014). Sie beinhaltet sowohl die explizite Friedens- und Konfliktforschung als auch friedenswissenschaftlich relevante Forschungsstränge der Internationalen Beziehungen (IB), der Sicherheitsstudien und der Aussenpolitikanalyse. ${ }^{1}$ Grundlage unserer Erhebung bildeten diejenigen Personen, Publikationen und Institutionen, von denen wir aufgrund einer Literaturrecherche und unseres breiten Netzwerkes Kenntnis hatten. ${ }^{2}$ Damit ist kein Anspruch auf Vollständigkeit verbunden. Wir hoffen aber, einen repräsentativen Überblick der in der Schweiz erbrachten wissenschaftlichen Leistungen in der Friedensforschung zu liefern und diese zugleich historisch und strukturell analysieren und kategorisieren zu können. Ziel ist es, diese Forschung im internationalen Vergleich darzustellen und das besondere Profil der Schweizer Friedensforschung herauszuarbeiten. Hierbei gehen wir auch auf das Verhältnis der Friedensforschung zur Aussen- und Sicherheitspolitik der Schweiz und zum Konzept der Neutralität ein.

Der Artikel bietet zuerst eine Übersicht der historischen und institutionellen Entwicklung der Schweizer Friedensforschung. Danach zeigt er ihre disziplinäre, methodologische und thematische Vielfalt auf. In einem dritten Schritt untersucht er die internationale Einbettung der Schweizer Friedensforschung und ihre Spezifität. Dazu gehört insbesondere die gezielte Förderung von Nord-Süd-Partnerschaften. Der Artikel untersucht im Folgenden die Praxisnähe der Schweizer Friedensforschung und geht schliesslich noch auf ihre epistemologische Verortung ein. In den Schlussfolgerungen weisen wir auf Desiderata inhaltlicher und forschungspolitischer Natur hin.

\footnotetext{
${ }^{1}$ Dies betrifft Forschung mit einem geografischen Schwerpunkt ausserhalb der Schweiz. Allerdings betrachten wir nicht alle Forschenden, die zu Kontinenten mit bewaffneten Konflikten arbeiten, automatisch als Friedensforschende.

${ }^{2}$ Wir definierten als Schweizer Friedensforschung diejenige Forschung, die an einer Schweizer Institution betrieben wird. Die Nationalität der forschenden Person spielte keine Rolle.
} 


\section{Historische und institutionelle Entwicklung}

Während die explizite Friedensforschung erst relativ spät gegen Ende des Ost-WestKonfliktes begründet wurde, gab es in der Schweiz schon seit den 1920er-Jahren Forschungsstätten, in denen friedensrelevante Fragen bearbeitet wurden. Auch die Politikwissenschaft etablierte sich in der Schweiz erst in den 1970er-Jahren und damit im internationalen Vergleich eher spät.

Bereits die ersten Schritte der Friedensforschung erfolgten in der Schweiz in enger Anlehnung an die internationale und nationale Politik. Massgebend dafür waren die Entwicklung der Stadt Genf als Standort internationaler Organisationen seit der zweiten Hälfte des 19. Jahrhunderts und später insbesondere des Völkerbundes sowie die Neutralität als aussenpolitische Maxime. Mit den internationalen Agenturen wuchsen in Genf sowohl das Wissen wie auch der Wissensbedarf zur internationalen Zusammenarbeit. Leitende internationale Funktionäre wirkten als Dozierende in Forschungsstätten. Zugleich gaben sie diesen Forschungsstätten Studien zu verschiedenen Aspekten der internationalen Politik in Auftrag. Der Austausch internationaler Expert*innen mit Wissenschaftler*innen unterschiedlicher Herkunft lieferte einen fruchtbaren Humus für die Genese neuer Fragestellungen.

Mit dem Institut de hautes études internationales HEI (später: IHEID) entstand in den 1920er Jahren ein zunächst vorwiegend von Völkerrechtler*innen und Ökonom*innen geprägtes Forschungsinstitut, in welchem vor dem Hintergrund der ersten Gehversuche der kollektiven Sicherheit weltweit führende Überlegungen zur Eindämmung von Kriegen angestellt wurden. Politisch gesehen variierten die Ansichten der jeweiligen Wissenschaftler*innen beträchtlich, wie die Beispiele des im schweizerisch bürgerlich-konservativen Milieu verankerten William E. Rappard (1940) und des deutschstämmigen Pazifisten Hans Wehberg (1930) zeigen, die beide im damals noch von der Rockefeller Stiftung finanzierten Institut wirkten.

In engem Zusammenhang mit der kollektiven Sicherheit wurde die Neutralität der Schweiz diskutiert. Es ging vor allem um die Bedingungen, unter denen die Neutralität als vereinbar mit der kollektiven Sicherheit erachtet wurde (Schindler 1938; Guggenheim 1945). Bei allen institutionellen Unterschieden teilen beide Konzepte ihre Verankerung im Völkerrecht sowie ihre Skepsis gegenüber Kriegen als Mittel zur Lösung von Konflikten. Jenseits der Schweiz erfolgten damals in Verbindung beider Konzepte auch Überlegungen zur „kollektiven Neutralität“ (Cohn 1939).

Im Kalten Krieg wurden diese ersten Ansätze der Friedensforschung von der Forschung zur Sicherheitspolitik verdrängt. Zugleich gewann die Neutralität der Schweiz an Bedeutung: Letztere wurde ein privilegierter Ort für den Austausch zu Fragen des Ost-West-Konfliktes. In Genf entstanden neue Ausbildungsgänge, die wirtschaftlichen, politischen und sicherheitspolitischen Dimensionen der globalen Politik gewidmet waren. Der damalige Leiter des HEI, Jacques Freymond (1987), hatte zugleich die Leitung des Nationalen Forschungsprogramms ,Sicherheitspolitik" (NFP 11) inne. Auch war er Mitglied des Internationalen Komitees des Roten Kreuzes (IKRK) und in weiteren humanitären und wirtschaftlichen Organisationen und Verbänden engagiert. Im selben Zeitraum wurden am HEI und an der Eidgenössischen Technischen Hochschule Zürich (ETHZ) zwei Lehrstühle für Sicherheitspolitik neu besetzt, aus denen zwei Forschungszentren entstanden: das Programm für 
strategische und internationale Sicherheitsstudien (PSIS) in Genf und das Zentrum für Sicherheitsstudien (CSS) in Zürich. Curt Gasteyger (1986), von der Ausbildung her Jurist, setzte sich am HEI primär mit Fragen der internationalen Sicherheit und der Rüstungspolitik auseinander. Er war auf den Amerikaner Louis J. Halle gefolgt, der wohl als erster in der Schweiz Sicherheitspolitik unterrichtet hatte. Kurt Spillmann (1984) aus Zürich war als Historiker ein Experte amerikanischer Aussen- und Sicherheitspolitik. Trotz Fokus auf sicherheitspolitische Fragen wurde im Umfeld dieser Forschungsstellen die Grundlage für die moderne Friedensforschung in der Schweiz gelegt.

An der Universität Zürich forschte der Politikwissenschaftler Daniel Frei zu grundsätzlichen Fragen internationaler Konflikte und Kooperation, zu spezifischen Aspekten von Gewalt und Frieden sowie zum Risiko eines unbeabsichtigten Nuklearkrieges (Frei 1977, 1982, 1983a, 1983b). Mit Günther Baechler und Heinz Krummenacher waren beim CSS zwei Wissenschaftler tätig, die zugleich massgeblich zur Gründung der Schweizerischen Friedensstiftung (später: swisspeace) im Jahre 1988 beitrugen. Während Baechler sich vor allem im Bereich der Forschung zu Umweltkonflikten einen Namen machte (z.B. Baechler et al. 2002), zeichnete sich Krummenacher durch seine Forschung zur Risikoanalyse und zur politischen Frühwarnung aus (z. B. Krummenacher und Schmeidl 2001). Laurent Goetschel, der etwas später zu swisspeace stiess, kam ursprünglich vom PSIS und forschte damals zur Aussenpolitik der Schweiz und zur Neutralität (Goetschel 1999; Goetschel et al. 2002).

Die Gründung der Schweizerischen Friedensstiftung erfolgte 1988 nach langjährigen politischen Diskussionen (swisspeace 2008). Ihr Stiftungszweck beinhaltet, ,die schweizerische Friedens- und Sicherheitspolitik in Forschung, Konzeption, Ausbildung, Beratung und Kommunikation mitzugestalten sowie bei ihrer Verwirklichung mitzuwirken“ (swisspeace 2012). Rund sechzig Jahre später knüpfte die Stiftung damit an die Tradition des HEI aus der Zwischenkriegszeit an. Dies betrifft sowohl den Inhalt der Forschung als auch ihr Ziel, Auswirkungen auf die relevante politische Praxis zu haben. Seit 2010 ist swisspeace ein assoziiertes Institut der Universität Basel.

Nach dem Ende des Ost-West-Konfliktes entstanden in kurzer Abfolge die drei sogenannten „Genfer Zentren“: Geneva Centre for Security Policy (GCSP), Geneva International Centre for Humanitarian Demining (GICHD) und Geneva Centre for the Democratic Control of Armed Forces (DCAF). Vor allem das GCSP erbte internationale Ausbildungsprogramme, die während des Kalten Krieges am HEI entstanden waren. Rechtlich handelt es sich bei allen drei Zentren um Stiftungen, deren Mitglieder Staaten sind. Während sich das GICHD vorwiegend operationell engagiert, ist das GCSP primär im Ausbildungsbereich tätig und das DCAF auf internationale Programme zu Fragen der Sicherheitssektor-Reform (SSR) spezialisiert. Die drei Zentren sind zusammen mit dem heutigen Institut de hautes études internationales et de développement (IHEID) ${ }^{3}$ im ,Maison de la Paix“ in Genf untergebracht. Sie sind jedoch keine eigentlichen Forschungsinstitutionen - anders als die IHEID eigenen

\footnotetext{
${ }^{3}$ Dieses entstand im Jahre 2007 aus der Fusion des IUED, dem Institut für Entwicklungsstudien, und des HEI.
} 
Forschungszentren wie zum Beispiel das Global Governance Centre und das Centre on Conflict, Development and Peacebuilding (CCDP). Daneben bietet die 2007 von der Universität Genf und dem IHEID gegründete Genfer Akademie für humanitäres Völkerrecht und Menschenrechte Nachdiplomstudiengänge und Weiterbildung für Führungskräfte an und betreibt Forschung zum humanitären Völkerrecht und dem Schutz der Menschenrechte in bewaffneten Konflikten.

Dieser kurze historische Überblick zeigt, dass die institutionelle Entwicklung der Friedensforschung in der Schweiz in engem Bezug zur Entwicklung der internationalen Politik erfolgte. Es waren die ersten Bestrebungen, die kollektive Sicherheit zu institutionalisieren, welche in der Zwischenkriegszeit zur Gründung des Genfer Instituts für internationale Studien führten. Wie damals üblich waren vor allem Historiker*innen, Ökonom*innen und Völkerrechtler*innen wissenschaftlich gefragt. Mit dem Kalten Krieg hielten zunächst politikwissenschaftliche Realisten im Bereich der Sicherheitspolitik Einzug. Die neu gebildeten Forschungseinheiten standen thematisch und personell wiederum in regem Austausch mit der politischen Praxis. Die Neutralität der Schweiz begünstigte die Einrichtung von Lehr- und Ausbildungsprogrammen, welche Expert*innen aus allen Weltgegenden zusammenbrachten.

Das Ende des Ost-West-Konfliktes verlieh der Friedensforschung einen erneuten Schub. Dieser wurde durch das stärkere Engagement der offiziellen Schweiz im Bereich der internationalen Friedensförderung begünstigt (EDA 2006; Schweizerischer Bundesrat 2011; Graf und Lanz 2013). Mit swisspeace sollte die praxisorientierte Friedensforschung gefördert werden. Die drei Genfer Zentren entstanden teils als Erbe von Aktivitäten aus der Zeit des Kalten Krieges, teils vor dem Hintergrund des wachsenden Volumens an globalen Friedensförderungsprogrammen. Ihr klarer Fokus auf die Praxis entkoppelte sie jedoch von der Friedensforschung. Parallel dazu entstanden im Sog der Entwicklung der Politikwissenschaft und anderer Sozialwissenschaften an mehreren Universitäten neue Forschungsgruppen, die sich im Rahmen einzelner Lehrstühle mit Fragen der Friedensforschung befassten, in der Regel aber eine grössere Praxisferne aufwiesen.

\section{Disziplinäre, methodologische und thematische Vielfalt}

Als Folge der geschilderten Entwicklung war die Friedensforschung in der Schweiz stets an verschiedenen Institutionen und in unterschiedlichen Disziplinen vertreten. Dabei weist sie eine ausgesprochene disziplinäre, methodologische und thematische Vielfalt auf.

\subsection{Disziplinäre und methodologische Vielfalt}

Die Friedensforschung wird in der Schweiz von unterschiedlichen Disziplinen wie der Politikwissenschaft, Soziologie, Ethnologie, Geschichte, Geografie, Recht und Wirtschaft getragen, in einem geringeren Masse auch von der Psychologie, Ethik und Philosophie. Während sie an den Universitäten zumeist in der Politikwissenschaft beheimatet ist, zeichnen sich die Institute, die sich mit der Thematik befassen, durch eine stärkere Interdisziplinarität aus. 
In methodologischer Hinsicht kann eine grobe Kategorisierung vorgenommen werden, der zufolge in der Schweiz die Konfliktforschung eher auf quantitativer und die Friedensforschung eher auf qualitativer Grundlage erfolgt.

Quantitative Forschung wird von der von Lars-Erik Cederman geführten Forschungsgruppe International Conflict Research (ICR) am Center for Comparative and International Studies (CIS) der Universität Zürich und der ETHZ betrieben (Wucherpfennig et al. 2016; Bormann et al. 2017; Hunziker und Cederman 2017). Ausserdem wird am Lehrstuhl von Simon Hug an der Universität Genf auf quantitative Methoden zurückgegriffen (Hug und Wegmann 2013; Hug 2016; Bohnet et al. 2018). Am CSS kommen teilweise ebenfalls quantitative Methoden zum Einsatz (Nussio 2017; Duursma 2017, 2019). Momentan wird beispielsweise ein quantitativer Datensatz zu allen Bürgerkriegswaffenstillstandsabkommen seit 1989 aufgrund systematisch gesammelter und kodierter Daten zusammengestellt (Clayton und Sticher o.J.). Die von diesen Forschenden eingesetzten Methoden reichen von Modellierungen, über statistische Analysen, Umfragen, Ökonometrie, bis zu Computersimulationen.

Einen Schwerpunkt auf fallbasierter qualitativer Friedensforschung weist demgegenüber swisspeace auf (Bächtold 2015; Balthasar 2015, 2019; Santschi 2016; Bernath 2016; Hellmüller 2018). ${ }^{4}$ So wird zum Beispiel zur Rolle von Normen in der Mediation anhand dreier Fallstudien geforscht (Hellmüller et al. 2017), zum Aufbau von Resilienz in staatsbildenden Prozessen anhand von sechs Fallstudien (Balthasar 2019) und zu lokaler Justiz und traditionellen Autoritäten anhand einer Fallstudie (Santschi 2016). Auch am Global Governance Centre des Graduate Institutes greifen verschiedene Forschungsprojekte auf komparative Fallstudienanalysen zurück (Biersteker et al. 2016; Haftel und Hofmann 2017; Biersteker 2017). Im Projekt „UN Reform and Effectiveness“ werden sechs UN Agenturen und Organisationen untersucht. ${ }^{5}$ Ein anderes Projekt untersucht die Wirkung von gezielten UN-Sanktionen (Biersteker et al. 2018a). ${ }^{6}$ Am CCDP forscht Keith Krause seit vielen Jahren im Bereich der Critical Security Studies (Krause 2012, 2013). Am Zentrum für Afrikastudien der Universität Basel werden auch vorwiegend qualitative Fallstudien durchgeführt (Kesselring 2015; Koechlin und Förster 2016; Förster 2018). Qualitative Methoden, die in der Schweiz angewendet werden, umfassen komparative oder Einzelfallstudien, Diskurs- und Inhaltsanalysen, Ethnographien und Archivforschung.

\footnotetext{
${ }^{4}$ Vor rund zehn Jahren wurde ein zehnjähriges Programm zur Politischen Frühwarnung (FAST) eingestellt, das auf Ereignisdatenanalyse basierte (Gattiker et al. 2006).

5 Weltgesundheitsorganisation, Weltorganisation für geistiges Eigentum, Welthandelsorganisation, Internationaler Währungsfonds, UN Menschenrechtskommission/-Rat und die Internationale Arbeitsorganisation (siehe http://graduateinstitute.ch/home/research/centresandprogrammes/global-governance/researchprojects/un-reform-and-effectiveness.html).

${ }^{6}$ Siehe https://www.graduateinstitute.ch/research-centres/global-governance-centre/targeted-sanctionsinitiative.
} 


\subsection{Thematische Vielfalt}

Thematisch zeichnet sich die Friedensforschung in der Schweiz durch eine breite Palette an Forschungsgegenständen aus. Dies folgt aus ihrer historischen Entwicklung und der daraus resultierenden institutionellen Diversifizierung. Zudem sind die Friedensforschungsbeiträge vielfach in breitere methodologische oder geografische Forschungskontexte integriert.

Im Sinne einer groben thematischen Kategorisierung kann die schweizerische Friedensforschung in Friedensforschung, Konfliktforschung, IB-basierte Analysen und Aussenpolitikanalyse eingeteilt werden.

Die Friedensforschung versucht, ein besseres Verständnis der Instrumente, Prozesse, Akteure und Dynamiken zu schaffen, die helfen, Kriege zu beenden, Gewalt zu verhindern und friedliche Strukturen aufzubauen. Instrumente und Prozesse der Friedensförderung sind zum Beispiel Waffenstillstandsabkommen, Mediation und nationale Dialoge (Mason und Sguaitamatti 2011; Hellmüller et al. 2014; Lanz 2017; Clayton und Sticher o.J.). Auch zum Nexus von Sicherheit und Frieden (Krause und Jütersonke 2005), zur Rolle der Religion (Ullmann und Aung 2017) und Diversität (Hirblinger und Landau 2018) oder zu Übergangsjustiz und Vergangenheitsarbeit (Penic et al. 2016, 2017; Jones und Bernath 2017; Haldemann und Unger 2018) wird rege geforscht. Daneben ist die Forschung zu verschiedenen Akteuren, wie die Rolle von lokalen Akteuren in Friedensprozessen und insbesondere der Zivilgesellschaft und deren Partizipation in politischen Verhandlungen (Paffenholz 2010, 2014; Hellmüller und Zahar 2018; Hellmüller 2019), des Staates im Sinne der Staatlichkeit und Staatslegitimität (Milliken und Krause 2002; Hagmann und Péclard 2010; Balthasar 2018) und zu nicht-staatlichen bewaffneten Akteuren (Hagmann und Péclard 2011; Leander 2017; Mohamedou 2017a, 2017b; Kaplan und Nussio 2018) relativ gut vertreten. Einen interessanten Forschungszweig stellt das Thema des Zusammenspiels zwischen Wirtschaft und Frieden dar (Wennmann 2011, 2012; Graf und Iff 2016; Ganson und Wennmann 2016). Schliesslich ist das Gebiet der Analyse und Wirkung der Friedensförderung (Goetschel und Pfluger 2014) ein aufkommendes, aber noch auszubauendes, Forschungsthema.

Das Hauptziel der Konfliktforschung hingegen ist es, die Ursachen und Dynamiken, die zu Kriegen führen, besser zu verstehen. Zumindest implizit ist damit die Absicht verbunden, deren Aufkommen in Zukunft zu verringern. In der Schweizer Konfliktforschung wird ein bestimmtes Augenmerk auf inner- und zwischenstaatliche Konflikte und deren Ursachen gelegt. Vor allem prominent sind hier Forschungsstränge zur Rolle von ethnischen Gruppen und Ungleichheit in Konflikten, zu Mechanismen politischer Mobilisierung entlang ethnischer Linien und ethnischer Inklusion (Cederman et al. 2013, 2015; Vogt et al. 2016; Wucherpfennig et al. 2016; Bormann et al. 2017; Gohdes 2017). Gleichzeitig werden ökologische Faktoren als Konfliktursache analysiert (Spillmann und Bächler 1996; Krummenacher 2008). An der Universität Genf wird im Rahmen des Geneva Water Hub zur Rolle von Wasser in bewaffneten Konflikten geforscht. ${ }^{7}$ Daneben wird auch der Einfluss von Demokratisierung, Staatenbildungsprozessen und Nationalismus auf bewaffnete Konflikte

\footnotetext{
7 Siehe https://www.unige.ch/gedt/recherches/chaire-unesco-en-hydropolitiques/.
} 
untersucht (Fleiner et al. 2003; Cederman et al. 2010, 2011; Macamo 2017). Auch verschiedene Konflikt-, Kriegsführungs- und Gewaltformen (Krause 2008; Daase und Davis 2015; Hayoz 2016; Cederman und Vogt 2017) sowie die Themen der Gewaltprävention (Austin und Bocco 2016), Gewaltreduktion in urbanen Kontexten (Hoelscher und Nussio 2016) und Rüstungstrends (Krause 2009, 2010, 2017) sind nennenswert. Ein wichtiger Forschungsstrang befasst sich auch mit den Folgen bewaffneter Konflikte. Insbesondere völkerrechtliche Studien zu Flüchtlingen und Binnenvertriebenen sind friedenswissenschaftlich zentral (Kälin 1991; Chetail 2011, 2016; Rüegger und Bohnet 2018).

Eine dritte Kategorie bilden Fragestellungen der Friedensforschung, die den Theorien der IB entspringen. Dazu gehören Fragen der globalen Gouvernanz (Cortell und Davis 1996; Davis 2012; Biersteker 2015) und insbesondere der Rolle der Vereinten Nationen, regionaler Organisationen und Nichtregierungsorganisationen (Haftel und Hofmann 2017; Andonova 2017). Auch die Auswirkungen von Sanktionen werden in der Perspektive der Friedensförderung untersucht (Hofmann und Yeo 2015; Biersteker et al. 2016, 2018b; Haftel und Hofmann 2017; Andonova 2017). Ausserdem stellen Normen einen Forschungsschwerpunkt dar, zum Beispiel das Zusammenspiel zwischen globalen und regionalen normativen Sicherheitsordnungen, Normen der Rüstungskontrolle oder die Rolle von Normen in Mediationsprozessen (Krause und Latham 1998; Krause 2002; Beyer und Hofmann 2011; Hofmann und Yeo 2015; Haftel und Hofmann 2017; Hellmüller et al. 2017).

Eine vierte Kategorie ist die Aussenpolitikanalyse. Hier bilden Analysen der Schweizer Friedenspolitik (Krummenacher 2006, 2011; Greminger 2011), der benachbarten Sicherheitspolitik (Mantovani 1999, 2015; Spillmann et al. 2001b; Wenger und Trachsler 2011; Hagmann et al. 2018) sowie vergleichende Studien zur Aussen- und Friedenspolitik verschiedener Europäischer Kleinstaaten (Wenger et al. 2006; Goetschel 2013) wichtige Bestandteile der Friedensforschung. Forschungsaktivitäten analog zu friedenspolitischen Prioritäten der Schweiz sind zum Beispiel im Bereich der Mediation oder der Vergangenheitsarbeit zu verzeichnen (Mason und Sguaitamatti 2011; Graf und Lanz 2013; Penic et al. 2016, 2017; Bernath et al. 2017). Weitere bearbeitete Subfelder weisen stärkere sicherheitspolitische Bezüge auf, wie etwa die Cybersicherheit (Dunn Cavelty 2008, 2018; Dunn Cavelty und Leese 2019), Nuklearwaffenpolitik (Popp und Wenger 2014) oder regionale und internationale Sicherheitsinstitutionen (Mantovani 1994; Hug und Wegmann 2013). Auch ein Teil der Forschung zur Menschenrechtspolitik der Schweiz kann der Friedensforschung zugeordnet werden (Kälin 1988; Gowlland-Debbas und Chetail 2002).

Ein alle Unterthemen übergreifendes Thema ist Gender und insbesondere die Genderdimension von sozialen Konflikten, bewaffneter Gewalt und der Friedensförderung (Zalewski et al. 2017; Prügl 2017).

In geografischer Hinsicht ist die Schweizerische Friedens- und Konfliktforschung breit abgestützt. Während ein gewisser Fokus auf Fallstudien aus Afrika zu verzeichnen ist (z. B. Südsudan, Somalia, Demokratische Republik Kongo, Burundi, Angola, Mozambique, Ghana, Elfenbeinküste, Nigeria, Guinea Conakry), wird auch zu Ländern in Asien (z. B. Myanmar, Indien, Indonesien), Europa (z. B. Ukraine, BosnienHerzegowina, Kosovo) und Lateinamerika (z. B. Kolumbien, Honduras, Guatemala) sowie zum Nahen Osten geforscht (Syrien, Ägypten, Israel/Palästina). Die meis- 
ten Friedensforschungsinstitute haben jedoch keinen ausdrücklichen geografischen Schwerpunkt, sondern arbeiten mit Fallstudien, die je nach Thema bestimmt werden.

Trotz dieser disziplinären, methodologischen und thematischen Vielfalt besteht in der Schweiz keine wahrzunehmende Kluft zwischen den verschiedenen Unterdisziplinen. So wird beispielsweise nicht streng zwischen Friedens- und Konfliktforschung unterschieden. Im Gegenteil, die meisten Friedensforschungsinstitutionen befassen sich trotz thematischem Fokus nicht ausschliesslich mit einem der vier Teilbereiche, sondern betrachten ausgewählte Kontexte oder Wissensgebiete aus unterschiedlichen Blickwinkeln.

\section{Internationale Einbettung}

Die Schweizer Friedensforschung ist ausgesprochen anschlussfähig an die internationale Forschung. Daraus resultiert eine starke internationale Verankerung. Die Einbettung in internationale Netzwerke und Teams manifestiert sich in gemeinsamen Workshops und Panels auf Konferenzen, aktiven Beteiligungen Schweizer Forschender in internationalen Gremien (z. B. in der Peace Studies Section der International Studies Association (ISA) oder der Standing Group zu Transitional Justice des European Consortium on Political Research (ECPR)), internationalen Kollaborationen in Forschungsprojekten und gemeinsamen Publikationen (z. B. Spillmann et al. 2001a; Jütersonke et al. 2009; Cederman et al. 2013; Daase und Davis 2015; Clayton und Thomson 2016; Kaplan und Nussio 2018). Regelmässig werden Schweizer Friedensforschende auch an ausländische Universitäten berufen.

Die internationale Vernetzung wird insbesondere im Rahmen der Nord-Süd-Forschung gezielt gefördert. Ein diesbezüglich wichtiges Instrument sind Forschungspartnerschaften. Diese unterstreichen die Bedeutung der Forschung mit Institutionen aus Ländern des globalen Südens, um das Wissen für den Umgang mit globalen Herausforderungen im Sinne der Ziele für eine nachhaltige Entwicklung (SDGs) zu verbessern. Die Kommission für Forschungspartnerschaften mit Entwicklungsländern (KFPE) entwickelte zwölf Prinzipien, deren Einhaltung helfen soll, auch bei Vorliegen grosser sozioökonomischer Unterschiede zwischen den Herkunftsländern der Wissenschaftler*innen gemeinsame Forschung zu ermöglichen. Damit werden die Voraussetzungen für einen kontinuierlichen und zielführenden Austausch der Forschenden mit den Gesellschaften in den jeweiligen Ländern verbessert (KFPE 1998, 2001).

Das vom Schweizerischen Nationalfonds (SNF) und der Direktion für Entwicklungszusammenarbeit (DEZA) gemeinsam finanzierte Förderungsinstrument Research on Global Issues for Development (r4d-Programm) unterstützt ebenfalls Forschungskollaborationen zwischen Forschenden in der Schweiz und in Entwicklungs- und Schwellenländern. So wurden globale Partnerschaften zu friedenspolitischen Fragen zwischen Forschenden in der Schweiz und in Ländern wie Ägypten, Elfenbeinküste, Ghana, Guatemala, Indien, Indonesien, Nigeria, Sambia und Südsudan ermöglicht. Geforscht wird zum Beispiel zu ethnischen Machtbeziehungen und Konflikten in fragilen Kontexten (Kapesa et al. 2016; Mukunto 2016; Vogt 2018), zur Wissensproduktion in der Friedensförderung (Palmer et al. 2015; Logo 2018) 
oder zur Gender-Dimension von sozialen Konflikten, bewaffneter Gewalt und der Friedensförderung (Zalewski et al. 2017; Rigual 2018).

Die über das r4d-Programm geförderte Forschung baut auf einer langjährigen Tradition im Bereich der Nachhaltigkeits- und Entwicklungsforschung auf. Dazu gehörte der Nationale Forschungsschwerpunkt Nord-Süd (NCCR North-South). In diesen Programmen wurde die Friedensforschung in die allgemeine Forschung zu NordSüd-Themen und zu den SDGs integriert. So wurden Friedens- und Konfliktaspekte der internationalen Zusammenarbeit (z. B. Verhältnis zu Armut, zu Gesundheit, zu Klima) untersucht. Auch hat sich die offizielle Schweiz stark für den Einbezug dieser Thematik in die internationale politische Agenda eingesetzt (SDG 16, Agenda 2030). Die erwähnten Programme sorgten dafür, dass sich die Friedensforschung in der Schweiz gegenüber der Forschung zu anderen Dimensionen globaler Politik bewähren musste. Zugleich förderten sie transdisziplinäre Ansätze. Dies führte einerseits zu einem intensiven Austausch zwischen Forschung und Praxis, andererseits aber zu einer teilweise intensiven Diskussion der Ergebnisse der Friedensforschung in der politischen Öffentlichkeit.

\section{Transdisziplinarität}

Die Schweizer Friedensforschung sieht sich bewusst als transdisziplinäre Wissenschaft. Sie wird selten als Grundlagenforschung gehandhabt, sondern vor allem in Departementen und Instituten betrieben, die mit Akteuren in der Friedensförderung und politischen Entscheidungsträger*innen kooperieren und Einfluss auf sie zu nehmen suchen. Es scheint einen Konsens darüber zu geben, dass die Friedensforschung per Definition praxisnah und -relevant sein sollte (Goetschel 2016) und dass ihr Potential Konflikte zu verhindern oder Gewalt zu reduzieren ein wichtiges Qualitätsmerkmal darstellt (Goetschel und Pfluger 2014). Dies betrifft sowohl die Friedens- und Konfliktforschung im engeren Sinn als auch den entsprechenden Bereich der IB und der Aussenpolitikanalyse.

Eine mögliche Erklärung für diese Praxisnähe liefert das Rollenkonzept der Neutralität, das seit der Entstehung der modernen Schweiz und ihrer Aussenpolitik in der zweiten Hälfte des 19. Jahrhunderts die zentrale Ausrichtung für die Aussenbeziehungen geliefert hat. Trotz variabler Anwendung in der politischen Praxis und unterschiedlichen wissenschaftlichen Einschätzungen bot dieses Konzept die wichtigste Grundlage sowohl für die Handhabung als auch für die Reflexion der Aussenpolitik der Schweiz (Kreis 2004; Rickli 2013). Zugleich haftet der Neutralität eine ausgeprägte friedenspolitische Dimension an: die Nichtteilnahme an militärischen Konflikten von Dritten ist ein Schritt weg von der Auseinandersetzung und damit hin zur Lösung des Konfliktes. Indem die Aussenbeziehungen der Schweiz während über 150 Jahren vor dem Hintergrund dieses Rollenkonzepts geführt wurden, war damit wissenschaftlich und praktisch immer eine Friedenskomponente enthalten. Anders als im Falle der anderen europäischen (neutralen) Kleinstaaten war dies keine konjunkturelle Erscheinung (bspw. durch den Ost-West-Konflikt bedingt), sondern sie gehörte zur aussenpolitischen Identität des Landes. Ein ähnliches Beispiel, 
wenn auch unter anderen Vorzeichen als der Neutralität, bietet die friedenspolitische Dimension der Aussenpolitik von Norwegen (Goetschel 2013).

Damit wies in der Schweiz die Aussenpolitikanalyse stets Bezüge zur Friedensforschung auf. Beispiele dafür lieferten Reflexionen zur Stärkung des Völkerrechts, zur Entwicklung der kollektiven Sicherheit, zur Rolle militärischer versus ziviler Konfliktlösung oder Interventionen, zur Anerbietung der Schweiz als Ort für internationale Verhandlungen oder zu den guten Diensten der Schweiz, die von der Wahrnehmung der Vertretung fremder Interessen bis hin zu modernen Formen der Mediation reichten (Stamm 1974; Fischer 2002). Letztere bietet eine gute Möglichkeit zur Illustration der Praxisnähe der Schweizer Friedensforschung. Wegen der Neutralitätspolitik ${ }^{8}$ der Schweiz werden ihre guten Dienste als Friedensvermittlerin regelmässig in Anspruch genommen. Mediation und Fazilitation sind deshalb zu Grundpfeilern der Schweizer Friedenspolitik geworden. Seit 2015 investiert das Eidgenössische Departement für auswärtige Angelegenheiten (EDA) gezielt in die Nachwuchsförderung und Professionalisierung der Mediation und finanziert entsprechende Forschungen und Weiterbildungsprogramme. Die Schweiz vermittelt direkt, agiert aber oft auch im Hintergrund und stellt Expertise zur Verfügung. Für beides arbeitet sie eng mit Forschungsinstituten zusammen. Im Jahre 2005 initiierte sie diesbezüglich das Mediation Support Project (MSP), gemeinsam durchgeführt von CSS und swisspeace. Nebst direkter Prozessunterstützung und Trainings unterstützt MSP das EDA mit Expertise und Analysen, die auf der Forschung der beiden Partnerinstitute aufbauen.

Die Praxisnähe wird zusätzlich durch die Ressortforschung des Bundes gefördert. Mit diesem Gefäss unterstützt die Schweizer Eidgenossenschaft Forschung zu für sie relevanten Themen. Meistens handelt es sich dabei um interdisziplinäre Forschungsvorhaben, welche die Grundlage für eine evidenzbasierte Politik bieten sollen. In der sicherheits- und friedenspolitischen Ressortforschung beinhalteten die im Jahre 2016 bestimmten Schwerpunktthemen unter anderem die Konfliktanalyse und -beilegung. Dabei soll die Forschung durch ein besseres Verständnis der Konfliktursachen, des humanitären Völkerrechtes und der Instrumente der Friedensförderung die Schweizer Sicherheits- und Friedenspolitik unterstützen. In der jüngeren Vergangenheit wurden zum Beispiel ein Mapping von Archiven in Friedens- und Versöhnungskommissionen sowie eine Studie zu mediationsunterstützenden Strukturen in Staaten, regionalen Organisationen und den Vereinten Nationen finanziert (Oliveira et al. 2014; Lanz et al. 2017).

Schliesslich sind Schweizer Friedensforschende auch immer wieder als Expert*innen unterwegs, sei dies für Friedensförderungsmandate des Aussenministeriums, als entsandte UN-Expert*innen oder als Trainer oder Coaches für Partner der Schweizer Friedensförderung.

\footnotetext{
${ }^{8}$ Daneben spielen die Mehrsprachigkeit, demokratische Struktur und die Abwesenheit einer kolonialen Vergangenheit eine zentrale Rolle.
} 


\section{Epistemologische Verortung}

Die Praxisnähe der Schweizer Friedens- und Konfliktforschung wirkt sich auf die Art des Wissens aus, die sie produziert. Hirsch Hadorn et al. (2008) unterscheiden drei Typen von Wissen: Erstens das Systemwissen, welches sich mit den Ursachen von Problemen, den darunterliegenden Strukturen und sozialen Prozessen sowie den Gründen und Dynamiken gewisser Prozesse befasst. Zweitens das Objektwissen, das sich mit Akteuren und ihren Rollen, Interessen, Optionen, Strategien und Notwendigkeiten für Wandel auseinandersetzt. Drittens das Transformationswissen, das die Informationen generiert, die benötigt werden, um das Handeln auf Programmund Projektebene zu beeinflussen. Die praktische Anwendbarkeit steigt von der ersten zur letzten Form des Wissens. In der Schweizer Friedensforschung sind alle drei Wissensformen präsent. Aufgrund ihrer Praxisnähe werden jedoch Objekt- und Transformationswissen stärker gewichtet.

Am eindeutigsten bei der Produktion von System- und allenfalls Objektwissen zu verorten, ist die Konfliktforschung. ${ }^{9}$ Am ICR steht beispielsweise bewusst nicht Praxisrelevanz der produzierten Erkenntnisse im Vordergrund, sondern deren akademische Rigorosität und Relevanz. Daneben gibt es Institutionen, die fast ausschliesslich Transformationswissen produzieren. Das DCAF beispielsweise hat eine der Praxis zugewandte Mission, in dem sie direkt SSR-Prozesse unterstützen und die nötige Expertise liefern. Sie beraten Partner, Regierungen und internationale Organisationen in SSR-Prozessen und geben rechtliche und politische Empfehlungen ab. Ein weiteres Beispiel ist das GCSP, das sich auf die Weiterbildung von Führungskräften im Gebiet der Sicherheitspolitik spezialisiert hat, und Raum für Dialoge und Politikanalysen bietet.

Institutionen wie das CSS der ETHZ oder swisspeace produzieren alle drei Arten von Wissen und deren Kombination ist Bestandteil ihrer Strategie. Das CSS ist sicherheitspolitisch orientiert und beschreibt seine Arbeit als „unabhängig, praxisrelevant und basierend auf einem soliden akademischen Fundament". ${ }^{10}$ swisspeace definiert sich als praxisorientiertes Friedensforschungsinstitut und will durch innovative Forschung Diskurse zur internationalen Friedenspolitik beeinflussen, neue Friedensförderungsinstrumente und -methoden entwickeln und anwenden und Akteure in der Friedensförderung unterstützen und beraten. ${ }^{11}$

In der Schweizer Friedensforschung etwas in den Hintergrund gerückt ist in den letzten Jahren das Systemwissen und vor allem derjenige Teil davon, der sich mit der Selbstreflexion (Weller 2017) befasst. Während die Selbstreflexion zur Zeit des Ost-West-Konfliktes und in den ersten Jahren danach überall dort, wo Friedensforschung betrieben wurde, zu deren unabdingbaren Bestandteilen gehörte, geriet sie mit der zunehmenden Selbstverständlichkeit der Forschung, aber vor allem auch in Anbetracht der friedenspolitischen Praxis in den Hintergrund.

\footnotetext{
9 Mit der Ausnahme des Small Arms Surveys, ein Exzellenzzentrum für die Generierung von evidenzbasiertem, unparteilichem und praxisrelevantem Wissen zu Handfeuerwaffen und bewaffneter Gewalt.

10 Siehe http://www.css.ethz.ch/en/center.html.

11 Siehe www.swisspeace.ch.
} 
Die ausgeprägte Praxisnähe eines grossen Teils der Schweizer Friedensforschung sorgte für eine grosse Nachfrage nach dem entsprechenden Objekt- und Transformationswissen. Im Sinne der gewollten Transdisziplinarität ist dies sehr wichtig. Zugleich erschwerte diese „Nähe am Objekt“ die Produktionsbedingungen für die kritische Reflexionsleistung. Während zuweilen bewusst der Nexus zwischen Theorie und Praxis untersucht und die Rolle der Friedensforschung auf einer Metaebene analysiert wird (Goetschel und Hagmann 2009; Goetschel und Pfluger 2014; Palmer et al. 2015), bilden solche Studien eher die Ausnahme als die Regel.

Dabei wäre es gerade aufgrund der Praxisnähe der Schweizer Friedensforschung wichtig zu analysieren, ,welche Beiträge sie selbst durch ihre Beschreibungen und Konstruktionen zu bestimmten Weltbildern und Vorstellungen politischer Konflikte leistet" (Weller 2017, S. 176). In der Tat besteht die Gefahr, dass Forschende, die zu nahe an politischen Entscheidungsträger*innen sind, zu schlichten Handlanger*innen und Kommentator*innen aktueller Prozesse mutieren (Lepgold 1998). Es besteht ein Paradoxon, dass mit zunehmender politischer Relevanz der eigenen Forschung das Risiko steigt, in eingesessenen Strukturen und Denkschemata zu verharren. Zugleich können auf Seiten der Kontaktpartner in der politischen Praxis selbstkritische Äusserungen ihnen wohlbekannter Forschender Irritationen hervorrufen. Dabei kann die Reflexion der eigenen Erkenntnisbedingungen zwangsläufig nur durch Forschende geleistet werden, die sich selbst in diesen kritisierten Strukturen befinden und damit „Kinder ihrer Zeit“ sind (Weller 2017, S. 175).

Von besonderer Bedeutung ist die Reflexion darüber, welchen unbeabsichtigten Beitrag die Friedens- und Konfliktforschung allenfalls zu Konflikten leistet. Zentral dafür ist das Konzept der Konfliktsensibilität. Während es bereits in Ansätzen auf die Feldforschung angewendet wurde (Gabriel und Goetschel 2017), fehlt eine vertiefte Anwendung des Begriffs in der allgemeineren Friedensforschung, beispielsweise im Sinne „der Konsequenzen der konkreten wissenschaftlichen Konfliktanalyse für den weiteren Verlauf des analysierten Konflikts“ (Weller 2017, S. 177). Im Zeitalter der Digitalisierung wird die Dringlichkeit der Frage nach den vertretenen Perspektiven in der Friedensforschung weiter zunehmen.

\section{Schlussfolgerungen}

Die Friedensforschung blickt in der Schweiz auf eine lange Tradition zurück. Sie ist im Verhältnis zur Grösse der Politikwissenschaft stark und diversifiziert vertreten. Letzteres betrifft sowohl die disziplinäre, methodologische als auch die thematische Ausrichtung. Hervorstechende Merkmale sind eine starke Präsenz im quantitativen Bereich der Konfliktforschung, eine etablierte Praxis qualitativ verorteter Arbeiten und Forschungsprogramme mit wenigen Fallstudien sowie mehrere Institute, die sich mit wissenschaftlich qualifizierten Arbeiten an der Schnittstelle zwischen Universitäten und der friedens- und sicherheitspolitischen Praxis befinden, insbesondere swisspeace und CSS, in geringerem Ausmass GCSP, GICHD und DCAF. Während die beiden erstgenannten Institutionen eindeutige strukturelle Verbindungen zu Universitäten (Universität Basel, ETHZ) aufweisen, sind die drei Genfer Zentren als internationale und von Staaten getragene Stiftungen hinsichtlich ihrer wissenschaft- 
lichen Produktion klar dem Policy-Bereich zuzuordnen. Insgesamt zeichnet sich die Schweizer Friedens- und Konfliktforschung durch ihre Vielfalt, ihre ausgeprägte internationale wissenschaftliche Verankerung und zugleich einen vergleichsweise engen Austausch mit der politischen Praxis aus. Im Hintergrund lieferte die Neutralität als aussen- und sicherheitspolitische Maxime einen Teil des Nährbodens, auf welchem die Friedensforschung gedieh, aber vor allem auch die Diskussionskultur, welche der Transdisziplinarität der Schweizer Friedensforschung Vorschub leistete. Letztere ermöglichte es der Friedensforschung, ihren Platz als integralen Bestandteil der Forschung zu den SDGs zu finden.

Nichtsdestotrotz weist die Friedensforschung in der Schweiz auch Lücken und Desiderata auf. Inhaltlich fällt im Bereich der Politikwissenschaft die relativ schwache Vernetzung der Friedensforschung mit der in der Schweiz stark verbreiteten Demokratie- und Wahlforschung auf. Hier würden sich gerade im Hinblick auf den Nord-Süd-Kontext interessante Perspektiven eröffnen. Weiter sind Forschungsvorhaben zur Rolle von Wirtschaftsakteuren sowie zum Bezug zwischen humanitärer und Friedenspolitik noch relativ schwach vertreten. In allen Feldern würde gerade in der Schweiz eine Stärkung der jeweiligen Forschung naheliegen. Die Demokratieund Wahlforschung ist eines der etabliertesten Felder der Schweizer Politikwissenschaft. Zudem zählt die Schweiz im Bereich der ausländischen Direktinvestitionen, als internationaler Handelsplatz und als Sitz multinationaler Unternehmen zu den weltweit wichtigsten Akteuren. Und schliesslich ist die Schweiz im humanitären Feld sowohl als Akteur als auch Gastgeberin von Organisationen wie dem IKRK oder Médecins sans Frontières (MSF) oder von UN-Agenturen wie dem UNHCR oder UNOCHA an vorderster Front unterwegs. Allgemein fehlt es aber in allen drei Feldern an Erkenntnissen über deren Auswirkungen auf Friedens- und Konfliktsituationen.

Weitere Desiderata gibt es im Bereich der Forschungsförderung: Institutionell ist die Schweizer Friedensforschung mit den erwähnten Forschungsinstituten und Zentren zwar gut vertreten, jedoch fehlen auf Seiten der Förderung jenseits der allgemeinen Forschung zur Nachhaltigkeit und den SDGs entsprechende Gefässe. Eine vergleichbare Institution zur Deutschen Stiftung Friedensforschung (DSF) wurde in der Schweiz nicht einmal andiskutiert. Auch auf der viel bescheideneren Ebene Nationaler Forschungsprogramme (NFPs) scheiterten in den vergangenen Jahren mehrere Vorstösse bereits in frühen Stadien. Ende 2017 wurde im Parlament sogar ein Vorstoss zur Streichung der Forschungssubvention an swisspeace nur knapp abgelehnt. ${ }^{12}$

Die Schweizer Friedensforschung vermittelt somit einen durchaus facettenreichen Gesamteindruck: disziplinär sowohl methodologisch und thematisch äusserst divers und praxisnahe, zugleich international kompetitiv und bestens verankert, institutionell jedoch weder als Gesamtheit präsent noch explizit gefördert, ja punktuell sogar in Frage gestellt. Es klafft somit eine Lücke zwischen dem Leistungsausweis der Schweizer Friedensforschung und ihrem Platz in der Förderlandschaft des Forschungsplatzes. Dieser strotzt nur so von Instrumenten und Programmen zur

${ }_{12}$ Siehe https://www.parlament.ch/de/ratsbetrieb/amtliches-bulletin/amtliches-bulletin-die-verhandlung en?SubjectId=42026. 
Generierung von Innovationen. Auch die Friedensforschung könnte ein paar davon gebrauchen.

Danksagung Wir danken Jamie Pring für ihre Unterstützung bei der Literatursuche, Dana Landau und David Lanz für ihre kritische Durchsicht und die bedeutsamen Ergänzungen sowie den anonymen Gutachtenden für ihre äusserst wertvollen Anregungen und Hinweise.

\section{Literatur}

Andonova, Liliana B. 2017. Governance entrepreneurs: international organizations and the rise of global public-private partnerships. Cambridge: Cambridge University Press.

Austin, Jonathan Luke, und Riccardo Bocco. 2016. Becoming a torturer: towards a global ergonomics of care. International Review of the Red Cross 98(903):859-888.

Bächtold, Stefan. 2015. The rise of an anti-politics machinery: peace, civil society and the focus on results in Myanmar. Third World Quarterly 36(10):1968-1983.

Baechler, Günther, Kurt R. Spillmann, und Mohamed Suliman. 2002. Transformation of resource conflicts. Approach and instruments. Frankfurt a.M., Bern: Peter Lang.

Balthasar, Dominik. 2015. From hybridity to standardization: rethinking state-making in contexts of fragility. Journal of Intervention and Statebuilding 9(1):26-47.

Balthasar, Dominik. 2018. State making in Somalia under Siyad barre: scrutinizing; historical amnesia and normative bias. International Journal of African Historical Studies 51(1):141-162.

Balthasar, Dominik. 2019. On the (in)compatibility of peace-building and state-making: evidence from Somaliland. The Journal of Development Studies 55(4):457-472.

Bernath, Julie. 2016. 'Complex political victims' in the aftermath of mass atrocity: reflections on the Khmer rouge tribunal. The International Journal of Transitional Justice 10(1):46-66.

Bernath, Julie, Adou Djané Dit Fatogoma, und Briony Jones. 2017. Understanding resistance to transitional justice. In Alternative approaches in conflict resolution, Hrsg. Christine Schliesser, Martin Leiner, 47-60. Cham: Palgrave Macmillan.

Beyer, Jessica L., und Stephanie Hofmann. 2011. Varieties of neutrality: norm revision and decline. Cooperation and Conflict 46(3):285-311.

Biersteker, Thomas. 2015. Global governance. In On governance: national and international dimensions of measuring governance effectiveness, Hrsg. Robert Rotberg. Waterloo: CIGI.

Biersteker, Thomas. 2017. Connecting scholarly expertise to international policy practice at the united nations. In The politics of expertise in international organizations, Hrsg. Annabelle Littoz-Monnet, 111-127. New York, London: Routledge.

Biersteker, Thomas, Rebecca Brubaker, und David Lanz. 2018a. UN sanctions: liability or asset in mediation processes? Geneva: Centre for Humanitarian Dialogue.

Biersteker, Thomas, Sue E. Eckert, und Marcos Tourinho. 2016. Targeted sanctions: the impact and effectiveness of UN action. Cambridge: Cambridge University Press.

Biersteker, Thomas, Sue E. Eckert, Marcos Tourinho, und Zuzana Hudáková. 2018b. UN targeted sanctions Datasets (1991-2013). Journal of Peace Research 55(3):404-412.

Bohnet, Heidrun, Fabien Cottier, und Simon Hug. 2018. Conflict-induced IDPs and the spread of conflict. Journal of Conflict Resolution 62(4):691-716.

Bormann, Nils-Christian, Lars-Erik Cederman, und Manuel Vogt. 2017. Language, religion, and ethnic civil war. Journal of Conflict Resolution 61(4):744-771.

Brühl, Tanja. 2012. Friedensforschung als ,Superwissenschaft‘ oder Sub-Disziplin? Zeitschrift für Internationale Beziehungen 19(1):171-183.

Brzoska, Michael. 2012. Friedensforschung und Internationale Beziehungen - Lob der Verschiedenheit. Zeitschrift für Internationale Beziehungen 19(1):127-141.

Cederman, Lars-Erik, und Manuel Vogt. 2017. Dynamics and logics of civil war. Journal of Conflict Resolution 61(9):1992-2016.

Cederman, Lars-Erik, T. Warren Camber, und Didier Sornette. 2011. Testing Clausewitz: nationalism, mass mobilization, and the severity of war. International Organization 65(4):605-638.

Cederman, Lars-Erik, Simon Hug, und Lutz Krebs. 2010. Democratization and civil war: empirical evidence. Journal of Peace Research 47(4):377-394.

Cederman, Lars-Erik, Kristian Skedre Gledtisch, und Simon Hug. 2013. Elections and ethnic civil war. Comparative Political Studies 46(3):387-417. 
Cederman, Lars-Erik, Nils B. Weidmann, und Nils-Christian Bormann. 2015. Triangulating horizontal inequality: Toward improved conflict analysis. Journal of Peace Research 52(6):806-821.

Chetail, Vincent. 2011. Théorie et pratique de l'asile en droit international classique: étude sur les origines conceptuelles et normatives du droit international des réfugiés. Revue générale de droit international public 115(3):625-652.

Chetail, Vincent. 2016. Looking beyond the rhetoric of the refugee crisis: the failed reform of the common European asylum system. European Journal of Human Rights 5:584-601.

Clayton, Govinda, und Andrew Thomson. 2016. Civilianizing civil conflict: civilian defense militias and the logic of violence in intrastate conflict. International Studies Quarterly 60(3):499-510.

Clayton, Govinda, und Valerie Sticher. forthcoming. Is the Devil in the Details? Ceasefire Agreements and Termination of Civil War.

Cohn, Georg. 1939. Neo-neutrality. New York: Columbia University Press.

Cooper, Robin, und Laura Finley. 2014. Introduction: exploring qualitative approaches to researching peace and conflict. In Peace and conflict studies research: a qualitative perspective, Hrsg. Robin Cooper, Laura Finley, 1-22. Charlotte: Information Age Publishing.

Cortell, Andrew P., und James W. Davis. 1996. How do international institutions matter? The domestic impact of international rules and norms. International Studies Quarterly 40(4):451-478.

Daase, Christopher, und James W. Davis. 2015. Clausewitz on small war. Oxford: Oxford University Press.

Davis, James W. 2012. A critical view of global governance. Swiss Political Science Review 18(2):272-286.

Dunn Cavelty, Myriam. 2008. Cybersecurity in Switzerland. Cham: Springer.

Dunn Cavelty, Myriam. 2018. Cybersecurity research meets science and technology studies. Politics and Governance 6(2):22-30.

Dunn Cavelty, Myriam, und Matthias Leese. 2019. Politicising security at the boundaries: privacy in surveillance and Cybersecurity. European Review of International Studies 5(3):49-69.

Duursma, Allard. 2017. Counting deaths while keeping peace: an assessment of the JMAC's field information and analysis capacity in Darfur. International Peacekeeping 24(5):823-847.

Duursma, Allard. 2019. Obstruction and intimidation of peacekeepers: How armed actors undermine civilian protection efforts. Journal of Peace Research 56(2):234-248.

Eidgenössisches Departement für auswärtige Angelegenheiten (EDA). 2006. Frieden und Menschenrechte in der schweizerischen Aussenpolitik. Bericht 2006 über Massnahmen zur zivilen Konfliktbearbeitung und Menschrechtsförderung. Bern: Eidgenössisches Departement für auswärtige Angelegenheiten (EDA). Genehmigt vom Bundesrat am 15. Juni 2017.

Fischer, Thomas. 2002. Switzerland's good offices: a changing concept, 1945-2002. Zürich: Forschungsstelle für Internationale Beziehungen / ETH Zürich.

Fleiner, Thomas, Walter Kälin, Wolf Linder, und Cheryl Saunders. 2003. Federalism, Decentralisation and conflict management in Multicultural societies. In Federalism in a changing world-learning from each other, Hrsg. Raoul Blindenbacher, Arnold Koller, 297-315. Montreal, Kingston: McGillQueen's University Press.

Förster, Till. 2018. La paix dans une zone de guerre. Lire la crise ivoirienne sur le temps long et par le bas. Politique Africaine 148(4):109-129.

Frei, Daniel. 1977. Friedensforschung und Friedenspolitik: Was trägt die Schweiz dazu bei? Zürich: Schweizerisches Institut für Berufspädagogik.

Frei, Daniel. 1982. Internationale Zusammenarbeit. Theoretische Ansätze und empirische Beiträge. Königstein: A. Hain.

Frei, Daniel. 1983a. Der ungewollte Atomkrieg. Eine Risiko-Analyse. München: Beck.

Frei, Daniel. 1983b. Friedenssicherung durch Gewaltverzicht? Eine kritische Überprüfung alternativer Verteidigungskonzepte. Bern: Schweizerisches Ost-Institut.

Freymond, Jacques. 1987. Ernstfall Frieden. Gedanken zur schweizerischen Sicherheitspolitik. Bern: Benteli Verlag.

Ganson, Brian, und Achim Wennmann. 2016. Business and conflict in fragile states: the case for pragmatic solutions. London: International Institute for Strategic Studies.

Gasteyger, Curt. 1986. International security and the arms race. Genf: PSIS.

Gattiker, Regula, August Hämmerli, und Reto Weyermann. 2006. Conflict and cooperation in an actors“ network of Chechnya based on event data. Journal of Conflict Resolution 50(2):159-175.

Goetschel, Laurent. 1999. Neutrality, a really dead concept? Cooperation and Conflict 34(2):115-139.

Goetschel, Laurent. 2013. Bound to be peaceful? The changing approach of Western European small states to peace. Swiss Political Science Review 19(3):259-278.

Goetschel, Laurent. 2016. Why peace research contributes to the relevance of IR. International Studies Association Annual Convention, Atlanta. 
Goetschel, Laurent, und Tobias Hagmann. 2009. Civilian Peacebuilding: peace by bureaucratic means? Conflict, Security \& Development 9(1):55-73.

Goetschel, Laurent, und Sandra Pfluger. 2014. Assessing quality in peace research. swisspeace Working Paper, Bd. 7/2014, 54-60. Bern: swisspeace.

Goetschel, Laurent, Magdalena Bernath, und Daniel Schwarz. 2002. Schweizerische Aussenpolitik: Grundlagen und Möglichkeiten. Schlussbericht des Nationalen Forschungsprogramms „Schweizerische Aussenpolitik“. Zürich: Verlag Neue Zürcher Zeitung.

Gohdes, Anita R. 2017. Bringing perpetrator and victim identities into empirical ethnic conflict research. Ethnopolitics 16(1):28-33.

Gowlland-Debbas, Vera, und Vincent Chetail. 2002. Switzerland and the international protection of refugees. The Hague, London, New York: Kluwer Law International.

Graf, Andreas, und Andrea Iff. 2016. Respecting human rights in conflict regions: how to avoid the "conflict spiral'. Business and Human Rights Journal 2(1):1-25.

Graf, Andreas, und David Lanz. 2013. Conclusions: Switzerland as a paradigmatic case of small-state peace policy? Swiss Political Science Review 19(3):410-423.

Greminger, Thomas. 2011. Swiss civilian peace promotion: assessing policy and practice. Zürich: Center for Security Studies (CSS) ETH Zürich.

Guggenheim, Paul. 1945. Der Völkerbund, Dumbarton Oaks und die schweizerische Neutralität. Zürich, New York: Europa Verlag.

Haftel, Yoram Z., und Stephanie Hofmann. 2017. Institutional authority and security cooperation within regional economic organizations. Journal of Peace Research 54(4):484-498.

Hagmann, Tobias. 2014. Revisiting peace and conflict studies. In Challenges of peace research, swisspeace working paper, Hrsg. Laurent Goetschel, Sandra Pfluger, 7-15. Bern: swisspeace.

Hagmann, Tobias, und Didier Péclard. 2010. Negotiating statehood: dynamics of power and domination in africa. Development and Change 41(4):539-562.

Hagmann, Tobias, und Didier Peclard. 2011. Negotiating statehood. Dynamics of power and domination in Africa. Oxford: Wiley-Blackwell.

Hagmann, Jonas, Stephan Davidshofer, Amal Tawfik, Andreas Wenger, und Lisa Wildi. 2018. The programmatic and institutional (re-)configuration of the Swiss national security field. Swiss Political Science Review 24(3):215-245.

Haldemann, Frank, und Thomas Unger. 2018. The united nations principles to combat impunity: a commentary. Oxford: Oxford University Press.

Hayoz, Nicolas. 2016. Organizing war and the military in society: a systemic perspective. Russian Sociology Review 15(2):9-24.

Hellmüller, Sara. 2018. Partners for peace: the interaction between local and international peacebuilding actors. Cham: Palgrave Macmillan.

Hellmüller, Sara. 2019. Beyond buzzwords: civil society inclusion in mediation. In Conflict management and transformation: theory and practice, Hrsg. Ho-Won Jeong, 47-63. Maryland: Rowman \& Littlefield.

Hellmüller, Sara, und Marie-Joëlle Zahar. 2018. Against the odds: civil society in the intra-Syrian talks. In Issue briefs. New York: International Peace Institute.

Hellmüller, Sara, Corinne Von Burg, und Mathias Zeller. 2014. Kann Mediation schaden? Konfliktsensitivität in internationalen Friedensverhandlungen. Konfliktdynamik 3(4):2-11.

Hellmüller, Sara, Julia Palmiano Federer, und Jamie Pring. 2017. Are mediators norm entrepreneurs? Bern: swisspeace.

Hirblinger, Andreas, und Dana M. Landau. 2018. Governing conflict: the politics of scaling difference. Journal of Intervention and Statebuilding 12(3):385-404.

Hirsch Hadorn, Gertrud, Holger Hoffmann-Riem, Susette Biber-Klemm, Walter Grossenbacher-Mansuy, Dominique Joye, Christian Pohl, Urs Wiesmann, und Elisabeth Zemp. 2008. The emergence of Transdisciplinarity as a form of research. In Handbook of Transdisciplinary research, Hrsg. Gertrud Hirsch Hadorn, Holger Hoffmann-Riem, Susette Biber-Klemm, Walter Grossenbacher-Mansuy, Dominique Joye, Christian Pohl, Urs Wiesmann, und Elisabeth Zemp, 19-39. Bern: Swiss Academies of Arts and Sciences.

Hoelscher, Kristian, und Enzo Nussio. 2016. Understanding unlikely successes in Urban violence reduction. Urban Studies 53(11):2397-2416.

Hofmann, Stephanie C., und Andrew I. Yeo. 2015. Business as usual: the role of norms in alliance management. European Journal of International Relations 21(2):377-401.

Hug, Simon. 2016. Dealing with human rights in international organizations. Journal of Human Rights 15(1):21-39. 
Hug, Simon, und Simone Wegmann. 2013. Ten years in the united nations: where does Switzerland stand? Swiss Political Science Review 19(2):212-232.

Hunziker, Philipp, und Lars-Erik Cederman. 2017. No extraction without representation: the Ethno-regional oil curse and secessionist conflict. Journal of Peace Research 54(3):365-381.

Jones, Briony, und Julie Bernath. 2017. Resistance and transitional justice. Abingdon, Oxon: Routledge.

Jütersonke, Oliver, Robert Muggah, und Dennis Rodgers. 2009. Gangs, Urban violence, and security interventions in Central America. Security Dialogue 40(4-5):373-397.

Kälin, Walter. 1988. Die Menschenrechtspolitik der Schweiz. Aussenpolitik, Schweizerisches Jahrbuch für politische Wissenschaft 28:185-207.

Kälin, Walter. 1991. Droit des réfugiés. Fribourg: Édition universitaire.

Kapesa, Robby, Jacob Mwitwa, und Donald Chikumbi. 2016. Social conflict in the context of the development of new mining concessions in Zambia. Southern African Peace and Security Studies 4(2):41-46.

Kaplan, Oliver, und Enzo Nussio. 2018. Explaining recidivism of ex-combatants in Colombia. Journal of Conflict Resolution 62(1):64-93.

Kesselring, Rita. 2015. Experiences of violence and the formation of the political : embodied memory and victimhood in South Africa. In The politics of governance: actors and articulations in Africa and beyond, Hrsg. Lucy Koechlin, Till Förster, 151-179. New York: Routledge.

KFPE. 1998. Swiss commission for research partnerships with developing countries: 11 principles. Bern: KFPE.

KFPE. 2001. Enhancing research capacity in developing and transition countries. Bern: KFPE.

Koechlin, Lucy, und Till Förster. 2016. The politics of governance: actors and articulations in Africa and beyond. New York: Routledge.

Krause, Keith. 2002. Multilateral diplomacy, norm building, and UN conferences: the case of small arms and lights weapons : review essay. Global governance 8(2):247-263.

Krause, Keith. 2008. New forms of violence. In The impenetrable fog of war: essays on modern war and strategic surprise, Hrsg. Patrick Cronin, 31-45. Westport: Praeger International Security.

Krause, Keith. 2009. Beyond definition: violence in a global perspective. Global Crime 10(4):337-355.

Krause, Keith. 2010. Armed groups and contemporary conflicts: challenging the Weberian state. London: Routledge.

Krause, Keith. 2012. Culture and security: multilateralism, arms control and security building. London: Frank Cass.

Krause, Keith. 2013. Critical perspectives on human security. In Routledge handbook of human security, Hrsg. Mary Martin, Taylor Owen, 96-113. London, New York: Routledge.

Krause, Keith. 2017. Small arms as a global public policy challenge: a view from a participant-observer. The Journal of Research Institute for the History of Global Arms Transfer 5(4):3-15.

Krause, Keith, und Oliver Jütersonke. 2005. Peace, security and development in post-conflict environments. Security Dialogue 36(4):447-462.

Krause, Keith, und Andrew Latham. 1998. Constructing non-proliferation and arms control: the norms of Western practice. Contemporary security policy 19(1):23-54.

Kreis, Georg. 2004. Kleine Neutralitätsgeschichte der Gegenwart. Bern: Haupt.

Krummenacher, Heinz. 2006. Alarmsignale der Gewalt. Politische Frühwarnung zwischen Friedensforschung und Friedenspolitik. Entwicklungspolitik 21:36-38.

Krummenacher, Heinz. 2008. Environmental factors as triggers for violent conflict: empirical evidence from the „FAST“ data base. In Monitoring environment and security. Bonn: International Center for Conversion.

Krummenacher, Heinz. 2011. Origins and state of the Art of Swiss peace policy. In The politics of peace: from ideology to pragmatism? Proceedings of the swisspeace 20th anniversary conference, Hrsg. Laurent Goetschel, 13-25. Berlin: LIT.

Krummenacher, Heinz, und Susanne Schmeidl. 2001. Practical challenges in predicting violent conflict. FAST: an example of a comprehensive early-warning methodology. Bern: Schweizerische Friedensstiftung.

Lanz, David. 2017. Ten recent developments in peace mediation. Perspektive Mediation 14(3):146-152.

Lanz, David, Jamie Pring, Corinne Von Burg, und Mathias Zeller. 2017. Understanding mediation support structures. Bern: swisspeace.

Leander, Anna. 2017. Digital/commercial (in)visibility: the politics of DAESH recruitment videos. European Journal of Social Theory 20(3):348-372.

Lepgold, Joseph. 1998. Is anyone listening? International relations theory and the problem of policy relevance. Political Science Quarterly 113(1):43-62. 
Logo, Kuyang. 2018. Exploring linkages of traditional and formal mechanism of justice and reconciliation in South Sudan. SSRN Electronic Journal.

Macamo, Elísio. 2017. Power, conflict, and citizenship: Mozambique's contemporary struggles. Citizenship Studies 21(2):196-209.

Mantovani, Mauro. 1994. Nato-Mitglied Schweiz? Voraussetzungen und Folgen einer sicherheitspolitischen Integration der Schweiz. Zürich: Forschungsstelle für Sicherheitspolitik und Konfliktanalyse, Eidgenössische Technische Hochschule.

Mantovani, Mauro. 1999. Schweizerische Sicherheitspolitik im Kalten Krieg 1947-1963. Zwischen angelsächsischem Containment und Neutralitäts-Doktrin. Zürich: Orell Füssli.

Mantovani, Mauro. 2015. Die Schweiz und die NATO vor der Partnerschaft für den Frieden (1949-1995). Politorbis 61:23-26.

Mason, Simon, und Damiano Sguaitamatti. 2011. Mapping Mediators: A comparison of third parties and implications for Switzerland. Zürich: Center for Security Studies.

Milliken, Jennifer, und Keith Krause. 2002. State failure, state collapse and state reconstruction: concepts, lessons and strategies. Development and Change 33(5):753-774.

Mohamedou, Mohammad-Mahmoud Ould. 2017a. A theory of ISIS: political violence and transformation of the global order. London: Pluto Press.

Mohamedou, Mohammd-Mahmoud Ould. 2017b. D'Al Qaïda à l'État islamique: acteurs non-étatiques mondialisés et évolution de la violence politique post-moderne. Relations internationales 172(4):3-14.

Mukunto, Ignatius Kabale. 2016. Zambia: Mediation and the transformation of the Lunda-Luvale conflict. Conflict Studies Quarterly 14:61-73.

Müller, Harald. 2012. Über allen Gipfeln ist Ruh: Zum Verhältnis von Friedensforschung und IB. Zeitschrift für Internationale Beziehungen 19(1):155-169.

Nussio, Enzo. 2017. The role of sensation seeking in violent armed group participation. Terrorism and Political Violence. Online First.

Oliveira, Ingrid, Briony Jones, und Rubli Rubli. 2014. Archives for a peaceful future: case descriptions. Bern: swisspeace.

Paffenholz, Thania. 2010. Civil Society and Peacebuilding. In Civil Society and Peacebuilding, Hrsg. Thania Paffenholz, 43-64. Boulder: Lynne Rienner Publishers.

Paffenholz, Thania. 2014. Civil society and peace negotiations: beyond the inclusion-exclusion dichotomy. Negotiation Journal 30(1):69-91.

Palmer, Nicola, Briony Jones, und Julia Viebach. 2015. Ways of knowing atrocity: a methodological enquiry into the formulation, implementation and assessment of transitional justice. Canadian Journal of Law and Society 30(2):173-182.

Penic, Sandra, Guy Elcheroth, und David Morselli. 2017. Intergroup forgiveness in the aftermath of symmetric and asymmetric communal violence: Contact density and nationalistic climates as contextual mediators. European Journal of Social Psychology 47(2):209-227.

Penic, Sandra, Guy Elcheroth, und Dario Spini. 2016. When is collective exposure to war events related to more acceptance of collective guilt? Journal of Conflict Resolution 62(1):143-173.

Popp, Roland, und Andreas Wenger. 2014. The origins of the nuclear nonproliferation regime. The International History Review 36(2):195-323.

Prügl, Elisabeth. 2017. Gender experts in international governance: mapping the contours of a field. In Expertes en genre et connaissances féministes sur le développement: Qui sait?, Hrsg. Christine Verschuur, 89-112. Paris: L'Harmattan.

Rappard, William E. 1940. The quest for peace. Cambridge: Harvard University Press.

Rickli, Jean-Marc. 2013. Neutrality Inside and Outside the EU: A Comparison of Austrian and Swiss Security Policies after the Cold War. In Small States in Europe. Challenges and Opportunities, Hrsg. Robert Steinmetz, Anders Wivel, 181-199. London: Routledge.

Rigual, Christelle. 2018. Rethinking the ontology of peacebuilding. Gender, spaces and the limits of the local turn. Peacebuilding 6(2):144-169.

Rüegger, Seraina, und Heidrun Bohnet. 2018. The Ethnicity of Refugees (ER): A new dataset for understanding flight patterns. Conflict Management and Peace Science 38(1):65-88.

Santschi, Martina. 2016. Encountering and "capturing" hakuma: negotiating statehood and authority in Northern Bahr El-Ghazal State, South Sudan. In Doctoral thesis University of Bern. Bern: University of Bern.

Schindler, Dietrich. 1938. Die Schweizerische Neutralität 1920-1938. Zeitschrift für ausländisches öffentliches Recht und Völkerrecht 8:413-444. 
Schweizerischer Bundesrat. 2011. Botschaft über die Weiterführung von Massnahmen zur Förderung des Friedens und der menschlichen Sicherheit 2012-2016. Bern: Schweizerischer Bundesrat.

Sidonia, Gabriel, und Laurent Goetschel. 2016. Motivation of philanthropists in peacebuilding: promoting peace as personal satisfaction? Journal of Peacebuilding \& Development 11(2):51-65.

Spillmann, Kurt R. 1984. Amerikas Ideologie des Friedens. Ursprünge, Formwandlungen und geschichtliche Auswirkungen des amerikanischen Glaubens an den Mythos von einer friedlichen Weltordnung. Bern, Frankfurt a.M.: Peter Lang.

Spillmann, Kurt R., und Günther Bächler. 1996. Kriegsursache Umweltzerstörung. Chur: Rüegger.

Spillmann, Kurt R., Thomas Bernauer, Jürg M. Gabriel, und Andreas Wenger. 2001a. Peace support operations: lessons learned and future perspectives. Bern: Peter Lang.

Spillmann, Kurt R., Andreas Wenger, und Christoph Breitenmoser. 2001b. Schweizer Sicherheitspolitik seit 1945. Zürich: Verlag Neue Zürcher Zeitung.

Stamm, Konrad Walter. 1974. Die guten Dienste der Schweiz. Aktive Neutralität zwischen Tradition, Diskussion und Integration. Bern: Peter Lang.

swisspeace. 2008. swisspeace 1988-2008. Bern: swisspeace.

swisspeace. 2012. Stiftungsurkunde. Bern: swisspeace.

Ullmann, Angela, und Seng Mai Aung. 2017. Addressing religion in conflict: insights from Myanmar. Journal for Human Rights and Peace Studies 3(2):129-158.

Vogt, Manuel. 2018. Ethnic stratification and the equilibrium of inequality: ethnic conflict in postcolonial states. International Organization 72(1):105-137.

Vogt, Manuel, Nils-Christian Bormann, und Lars-Erik Cederman. 2016. Democracy, ethnic exclusion, and civil conflict: the Arab spring revolutions from a global comparative perspective. In Peace and conflict, Hrsg. David Backer, Ravinder Bhavnani, und Paul Huth, 57-66. London: Routledge.

Wehberg, Hans. 1930. Die Ächtung des Krieges. Berlin: Vahlen.

Weller, Christoph. 2017. Friedensforschung als reflexive Wissenschaft: Lothar Brock zum Geburtstag. Sicherheit und Frieden (S+F) 35(4):174-178.

Wenger, Andreas, und Daniel Trachsler. 2011. Bulletin zur schweizerischen Sicherheitspolitik. Zürich: Center for Security Studies (CSS) ETH Zürich.

Wenger, Andreas, Victor Mauer, Bruno Stefano, Christiane Callsen, und Daniel Trachsler. 2006. Zivile Friedensförderung als Tätigkeitsfeld der Aussenpolitik. Zurich: Center for Security Studies / ETH Zürich.

Wennmann, Achim. 2011. Breaking the conflict trap? Addressing the resource curse in peace processes. Global governance 17(2):265-280.

Wennmann, Achim. 2012. The role of business in armed violence reduction and prevention. International Review of the Red Cross 85(886):919-940.

Wucherpfennig, Julian, Philipp Hunziker, und Lars-Erik Cederman. 2016. Who inherits the state? Colonial rule and Postcolonial conflict. American Journal of Political Science 60(4):882-898.

Zalewski, Marysia, Paula Drumond, Elisabeth Prügl, und Maria Stern. 2017. Sexual violence against men in global politics. London: Routledge. 